\title{
Care Transitions Program for High-Risk Frail Older Adults is Most Beneficial for Patients with Cognitive Impairment
}

\author{
Bjorg Thorsteinsdottir, MD 1,2,3,4*; Stephanie M Peterson ${ }^{5}$; James M Naessens, ScD'; Rozalina G McCoy, MD 1,2; \\ Gregory J Hanson, MD'; LaTonya J Hickson, MD2,7 Christina YY Chen, MD'; Parvez A Rahman²; Nilay D Shah, PhD; \\ Lynn Borkenhagen, PhD, CNP1; Anupam Chandra, MD'; Rachel Havyer, MD'1,2; Aaron Leppin, MD³; Paul Y Takahashi, MD'
}

${ }^{1}$ Division of Primary Care Internal Medicine, Mayo Clinic, Rochester, Minnesota; ${ }^{2}$ Center for the Science of Health Care Delivery, Mayo Clinic, Rochester, Minnesota; ${ }^{3}$ Knowledge and Evaluation Research Unit, Mayo Clinic, Rochester, Minnesota; ${ }^{4}$ Biomedical Ethics Research Program, Mayo Clinic, Rochester, Minnesota; ${ }^{5}$ Division of Biomedical Statistics and Informatics, Mayo Clinic, Rochester, Minnesota; ${ }^{6}$ Division of Health Care Policy and Research, Mayo Clinic, Rochester, Minnesota; 'Division of Nephrology and Hypertension, Mayo Clinic, Rochester, Minnesota.

BACKGROUND: Although posthospitalization care transitions programs (CTP) are highly diverse, their overall program thoroughness is most predictive of their success.

OBJECTIVE: To identify components of a successful homebased CTP and patient characteristics that are most predictive of reduced 30-day readmissions.

DESIGN: Retrospective cohort.

PATIENTS: A total of 315 community-dwelling, hospitalized, older adults ( $\geq 60$ years) at high risk for readmission (Elder Risk Assessment score $\geq 16$ ), discharged home over the period of January 1, 2011 to June 30, 2013.

SETTING: Midwest primary care practice in an integrated health system.

INTERVENTION: Enrollment in a CTP during acute hospitalization.

MEASUREMENTS: The primary outcome was all-cause readmission within 30 days of the first CTP evaluation. Logistic regression was used to examine independent variables, including patient demographics, comorbidities, number of medications, completion, and timing of program fidelity measures, and prior utilization of healthcare.
RESULTS: The overall 30-day readmission rate was $17.1 \%$. The intensity of follow-up varied among patients, with $17.1 \%$ and $50.8 \%$ of the patients requiring one and $\geq 3$ home visits, respectively, within 30 days. More than half $(54.6 \%)$ required visits beyond 30 days. Compared with patients who were not readmitted, readmitted patients were less likely to exhibit cognitive impairment $(29.6 \%$ vs $46.0 \% ; P=.03)$ and were more likely to have high medication use $(59.3 \%$ vs $44.4 \% ; P=.047)$, more emergency department (ED; 0.8 vs $0.4 ; P=.03$ ) and primary care visits (4.0 vs $3.0 ; P=.018$ ), and longer cumulative time in the hospital (4.6 vs 2.5 days; $P=.03$ ) within 180 days of the index hospitalization. Multivariable analysis indicated that only cognitive impairment and previous ED visits were important predictors of readmission.

CONCLUSIONS: No single CTP component reliably predicted reduced readmission risk. Patients with cognitive impairment and polypharmacy derived the most benefit from the program. Journal of Hospital Medicine 2019;14:329-335. Published online first February 20, 2019. (๐ 2019 Society of Hospital Medicine nplanned hospital admissions and readmissions have become a major focus of efforts to improve the value of healthcare given that these potentially preventable events exert substantial burden on patients, caregivers, health systems, and the economy. ${ }^{1}$ The percentage of patients who are rehospitalized within 30 days have decreased from $20 \%-21 \%$ at the start of the Accountable Care Act and readmission penalties to approximately $18 \% .^{2-5}$ Rehospitalization rates are $33 \%$ at 90 days and approach $40 \%$ at six months. ${ }^{6,7} \mathrm{Re}-$ admissions cost Medicare more than $\$ 26$ billion annually, ${ }^{4}$ with

*Corresponding Author: Bjorg Thorsteinsdottir, MD: E-mail: thorsteinsdottir. bjorg@mayo.edu; Telephone: 507-774-5944.

Received: July 19, 2018; Revised: October 18, 2018;

Accepted: October 21, 2018

() 2019 Society of Hospital Medicine DOI 10.12788/jhm.3112 one in five Medicare beneficiaries readmitted within 30 days of hospital discharge. ${ }^{8}$ Centers for Medicare and Medicaid Services and other payers use condition-specific and all-cause 30day unplanned readmission rates and potentially preventable admissions among patients with complex or multiple comorbidities for public reporting, value-based purchasing, and performance-based reimbursement. 9.10 Consequently, medical groups and hospitals have begun to place an increasing emphasis on improving the transitions of care following hospitalization with the goal of reducing unplanned readmissions. ${ }^{11}$ Care transitions programs have been shown to decrease readmission rates, mortality, and emergency department (ED) visits. ${ }^{12}$

Care transitions programs vary greatly in their scope of intervention and target groups, as well as in their efficacy in reducing readmissions. ${ }^{13,14}$ The Mayo Clinic Care Transition Program, hereafter referred to as CTP, was launched in 2011. This program was modeled after other successful programs and in- 


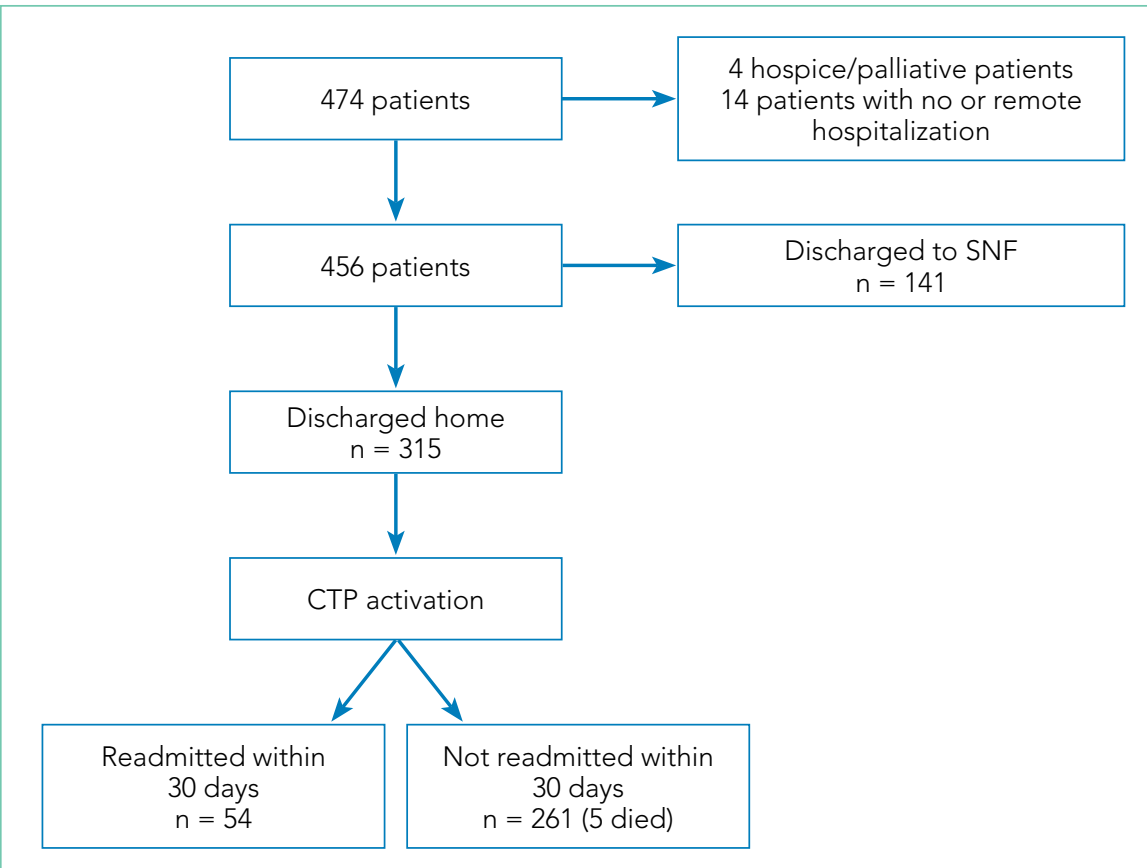

FIG 1. Derivation of the Cohort. Patients who were discharged to a SNF before CTP initiation were excluded.

Abbreviations: CTP care transitions program; SNF skilled nursing facility

volves home visits by a nurse practitioner (NP) and telephonic support and triage provided by a registered nurse (RN). It is offered to high-risk community-dwelling patients during their hospitalization and begins within a week of hospital discharge.

Although the CTP reduces 30-day readmissions from $20 \%$ to $17 \%,{ }^{7}$ it is a highly resource-intensive, multimodal, multidisciplinary program. Moreover, whether some components of the CTP are more critical than others remains unknown. Prior studies that examined the individual components of successful CTPs have suggested that a multipronged approach that includes close patient and caregiver support is most predictive of program efficacy. ${ }^{13}$ Long-term program sustainability would benefit from optimization of the most critical components of the program while reducing or eliminating resource-intensive factors that have negligible effects on program success. We therefore examined our CTP to identify whether and which program components are most critical for preventing 30-day readmissions and whether any patient characteristics contribute risk within this complex population.

\section{METHODS}

\section{Study Design and Setting}

This study is a retrospective cohort study of patients who were enrolled in the care transitions program of Mayo Clinic Rochester during the period January 1, 2010 to June 30, 2013. Patient demographic and clinical data were obtained from electronic health records (EHR), and information regarding CTP processes and interventions was obtained from a prospectively maintained program database. The study complied with the principles of the Declaration of Helsinki and was approved by the Mayo Clinic Institutional Review Board.

\section{Objectives}

The study aimed to describe the performance and utilization of a multidisciplinary care transitions program that has been successful in reducing readmissions for high-risk patients. The study also sought to identify patient and/or program factors associated with failure to prevent readmission within 30 days of program enrollment.

\section{Population}

Patients who were enrolled in the CTP following hospital discharge and seen for a posthospital in-home visit prior to hospital readmission (for those readmitted) were included. Patients discharged to a skilled nursing facility were excluded. Patients were eligible for CTP enrollment if they were hospitalized for any cause, community dwelling (including assisted living) prior to hospitalization, and $\geq 60$ years old with an Elder Risk Assessment (ERA) score $\geq 16$. $^{7}$ The ERA incorporates information regarding previous hospital days, age, and comorbid health burden and has been shown to predict 30-day readmissions, mortality, and critical illness (Figure 1). ${ }^{15,16}$

\section{Intervention}

Detailed descriptions of the CTP have been previously published.7,17 Patients meeting enrollment criteria are enrolled into the CTP by a RN prior to or immediately after hospital discharge. The patient is then seen at home within one to five business days of discharge and again the following week by a NP who performs medication reconciliation; chronic illness management; and acute illness, mobility, safety, and cognition assessments. The NP also provides patient education on self-care and advance care planning. Patient and caregiver support and liaisons with community resources are provided. Home visits by an NP or MD are continued as needed for at least one month. A RN case manager performs weekly phone calls to assess changes in the patient's clinical status and is available for phone triage of acute health issues. An interdisciplinary team composed of MDs, NPs, RNs, and pharmacists review patient management at weekly meetings. Although after-hours or weekend coverage for home visits are unavailable, an on-call primary care physician is available by phone at all times.

\section{Primary Outcome}

The primary outcome was all-cause hospital readmission within 30 days of the first CTP home visit, indicating successful program enrollment. Hospitalization was determined on the basis of billing codes from Mayo Clinic hospitals; this approach is $99 \%$ reliable in detecting readmissions for this population. ${ }^{18}$ 


\section{Secondary Outcome Measures}

Secondary outcome measures included six-month mortality and hospitalizations, as well as the number of hospital and ICU days and home, ED, primary care, and specialty office visits within 180 days after index hospitalizations as per the EHR. ED visits were counted only when they did not result in a hospital admission.

\section{Independent Variables}

Patient characteristics and clinical variables were retrieved from the EHR and included patient age, sex, and marital status. Comorbidities, ERA score, ${ }^{19}$ and Charlson comorbidity index $(\mathrm{CCl})^{20}$ within two years of program enrollment were determined by using ICD-9 billing codes. The frequencies of primary care and specialty visits within six months of the index hospitalization were also ascertained using the EHR. Mobility limitations and cognitive impairment were categorized as binary variables (yes/no) and were assessed at the first home visit by the NP. The presence of mobility limitations was defined as a Barthel's score of $<75^{21,22}$ or Timed up and Go time of $>20$ seconds. ${ }^{23}$ Cognitive impairment was established as Kokmen below the normal cutoff for patient's age group, ${ }^{24}$ Mini-Cog $\leq 2,{ }^{25}$ or AD8 $\geq 2 .{ }^{26}$ If these measures were not specifically documented during the first visit, clinical notes were queried for the description of pertinent cognitive and/or mobility limitations. Dementia diagnosis billing codes (ICD9 Code 290.*) were also included. High medication use was defined as $>14$ given the reported average medication number ranges from 8-13 in this population. ${ }^{27}$

As previously published, fidelity measures were abstracted from clinical notes by a trained nurse abstractor within 30 days of program enrollment and prior to a readmission. ${ }^{7}$ The five program fidelity measures included medication reconciliation, home service evaluation, advanced directives discussion, action plan for acute and chronic disease, safety plan, and discussion of community resources. The presence of advanced care planning was determined on the basis of visit medical notes and/or change of code status within the EHR, the identification or scanning of written advanced directives or "provider order for life-sustaining treatment," and documentation of the discussion of resuscitation status. It was abstracted in duplicate by a nurse abstractor with physician adjudication for disagreement. Moreover, whether the initial visit met the goal of being within five days of discharge was determined by using billing data.

\section{Analysis}

The contribution of each independent variable to 30-day readmission was first directly assessed by using a univariate logistic regression model. Five patients died within 30 days without being admitted. These deaths, however, were not censored given that home death (as opposed to hospital death) was considered a positive outcome of the CTP. Multivariable modeling was performed through log rank test with backwards elimination and included all independent variables with $P<$ .05. Variables with $P$ values between .05 and $>.1$ were tested for interaction with age and sex. Age was categorized as $<80$ or $\geq 80$ years. The length of hospital stay was categorized as $<3$ days (not qualifying for a Medicare skilled nursing facility), 3-13 days, or $\geq 14$ days.

This study had $30 \%$ power to detect a reduction of $5 \%$ in the rates of hospital admissions; $5 \%$ is the median absolute risk reduction reported by previous randomized studies on care transitions programs previously reported..$^{10}$ All analyses were performed using SAS 6.01 (SAS Inc., Cary, North Carolina).

\section{RESULTS}

\section{Study Population}

The study cohort included 315 patients who met the inclusion criteria (Fig 1). The demographic and clinical characteristics of the participants were ascertained at the time of CTP enrollment and are shown in Table 1. Patients were, on average, 82.5 (SD, 8.2) years old and had multiple comorbidities with a mean $\mathrm{CCl}$ score of 6.2 and ERA score of 18.5. Almost half of the patients (43.2\%) exhibited cognitive impairment and more than half (51.7\%) had mobility limitations. Among the patients, $42.9 \%$ had been hospitalized at least once in the 180 days prior to their CTP-qualifying hospitalization and $14.2 \%$ had $\geq 2$ hospitalizations prior to their CTP-qualifying hospitalization. Similarly, 32.4\% had at least one emergency department (ED) visit, and $3.5 \%$ had $\geq 3$ ED visits. The majority of patients had frequent outpatient visits, with $30.8 \%$ having $\geq 4$ office visits in primary care and $32.4 \%$ having $\geq 4$ specialty office visits in the preceding six months.

Readmissions, Mortality, ED, and Outpatient Visits Of the 315 patients, 54 (17.1\%) had a readmission within 30 days and seven (2\%) had $>1$ readmission. Among the patients, 126 (40.0\%) were readmitted at least once within 180 days with 55 (17.5\%) having more than one readmission. A total of 41 patients (13.1\%) died during the six-month follow-up period. The need for both office and ED visits was reduced compared to the 180 days prior to admission with the biggest difference in ED visits: 72 (22.9\%) of patients needed visits within 180 days of enrollment, as opposed to 102 (32.4\%) before enrollment.

\section{Impact of Patient Clinical Variables}

on Readmission Risk

Readmitted patients were less likely to exhibit cognitive impairment $(29.6 \%$ vs $46.0 \% ; P=.03)$ and were more likely to have high medication use $(59.3 \%$ vs $44.4 \% ; P=.047)$ than patients without readmission (Table 1). Readmitted patients had a higher frequency of visits to primary care (4.0 vs 3.0; $P=.02$ ) in the six months prior to admission and more hospital days in the prior year (4.6 vs $2.5 ; P=.04$ ) than those without readmission.

Multivariable analysis, which included the cognitive status of the patient; the high use of medication; and the number of ED visits, primary care visits, and hospital days in the previous six months, provided a $\mathrm{C}$ statistic of 0.665 . After backwards elimination, only the cognitive status of the patient and number of $E D$ visits remained predictive of readmission risk. 
TABLE 1. Baseline Demographics, Functional Status, and Comorbidity of Patients Discharged Home

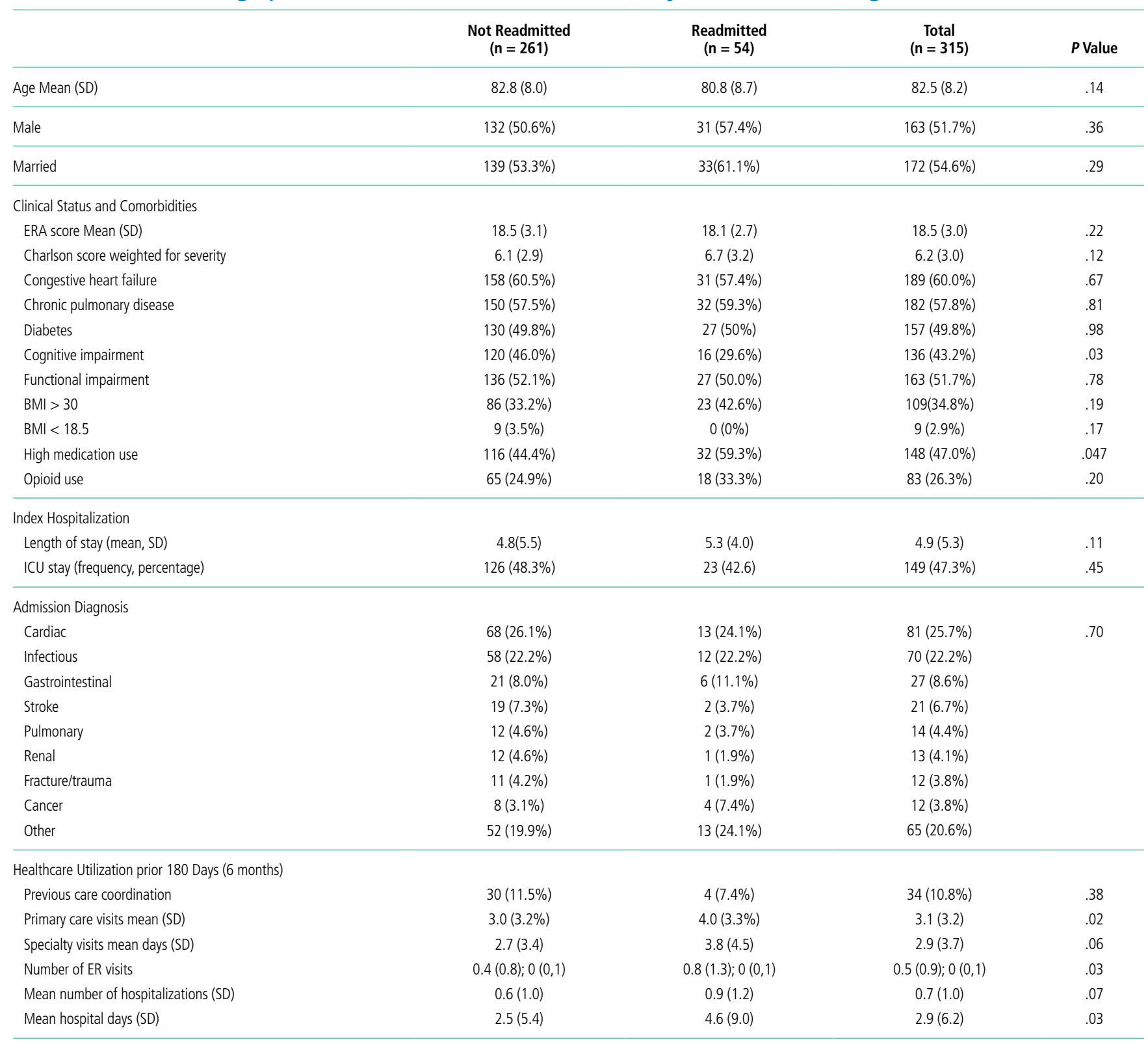

Abbreviations: BMI, body mass index; ERA, elder risk assessment; ICU, intensive care unit; SD, standard deviation.

Impact of Program Interventions on Readmission Risk

The completion of the CTP fidelity measures drastically varied with completion rates between 29.5\% (community resource evaluation) and $87.0 \%$ (home visit within five days of hospital discharge; Table 2). Only $12.1 \%$ of patients received all components of the CTP at the first home visit. Readmission rates among patients who received all program components (13.2\%) were lower than those among patients who did not receive all program components. This difference, however, failed to reach statistical significance. No single program component significantly reduced readmission risk. The completion rate of program fidelity measures increased with time (Figure 2). The present findings did not change even after performing sensitivity analysis that excluded the first program year. The overall agreement between chart abstractors on determining whether advance care planning occurred was $69.5 \%$ but the Cohens Kappa was only 18.4. This result was largely ascribed to the following: One abstractor counted the presence of a shorthand template used to document the delivery of an advance care planning document as discussion, whereas the other abstractor required further documentation or corroborating evidence (ie, change of code status). The majority of patients required multiple home visits to address ongoing medical needs (mean $2.7 ; \mathrm{SD}=1.3$ ) over the first 30 days. Among these patients, only $17.1 \%$ received one visit, and $54.6 \%$ of patients received $\geq 3$ 
TABLE 2. Fidelity Measures at First Home Visit and Home Visit within Five Days, Multivariable Analysis

\begin{tabular}{|c|c|c|c|c|c|}
\hline Fidelity Measures Achieved & $\begin{array}{l}\text { Number of Patients } \\
\text { with the Measure }\end{array}$ & $\begin{array}{l}\text { Readmission Rate } \\
\text { with Measure }\end{array}$ & $\begin{array}{l}\text { Readmission Rate } \\
\text { without Measure }\end{array}$ & Odds Ratio & $P$ Value \\
\hline Home visit within five days & $274(87.0 \%)$ & $17.2 \%$ & $17.1 \%$ & $1.01(.42,2.41)$ & .99 \\
\hline Safety discussion & $160(50.8 \%)$ & $15.6 \%$ & $18.7 \%$ & $0.81(.45,1.45)$ & .47 \\
\hline Community resource evaluation & $93(29.5 \%)$ & $15.1 \%$ & $18.0 \%$ & $0.81(.42,1.57)$ & .53 \\
\hline Action plan completed & $223(70.8 \%)$ & $19.7 \%$ & $10.9 \%$ & $2.02(.97,4.20)$ & .06 \\
\hline All pillars completed & $38(12.1 \%)$ & $13.2 \%$ & $17.7 \%$ & $0.71(0.26,1.90)$ & .49 \\
\hline
\end{tabular}

visits. Eleven (3.5\%) patients transitioned to a palliative homebound program that we began offering toward the end of this study to meet patient needs. ${ }^{28}$

\section{DISCUSSION}

The present study met our objective of identifying individual patient factors that are predictive of the success of our CTP. Cognitively impaired patients were less likely to be readmitted than cognitively intact patients. This finding is particularly important because patients with dementia constitute a subgroup that is at an increased risk of readmission after hospitalization ${ }^{29}$ and often suffer burdensome transitions at the end of life. ${ }^{30,31}$ High medication use and high number of visits to primary care and number of hospital days in the six months leading up to enrollment increase the likelihood of readmission and are plausible measures of disease severity or multi-morbidity that have been identified in previous studies. ${ }^{32,33} \mathrm{No}$ one program intervention was found to be significantly associated with readmission. This result is consistent with prior works that demonstrated the need for multifaceted and intensive interventions to reduce readmission risk among highly complex and multimorbid patients. ${ }^{13,14}$

Our findings suggest that the provision of an alternative to stressful hospitalization to cognitively impaired patients and their caregivers may be an important benefit of care transitions programs. Having a trusted team to consult in acute situations may have enabled early intervention and crisis avoidance. Avoiding hospitalizations and ED visits may also have been in line with their goals of care. ${ }^{34,35}$ Given that program intensity varied on the basis of the discretion of the clinical team, patients with cognitive impairment and their caregivers may also have received more intensive support than cognitively intact patients.

In contrast to recent systematic reviews, our study did not find that advance directive discussion had significant effects on reductions in readmission. ${ }^{36,37}$ The lack of discussion surrounding the goals of care for patients with serious illnesses was also listed as one of four factors that are strongly associated with preventability in a national cohort of readmitted general medicine patients. ${ }^{38}$ The lack of power and incomplete documentation may have contributed to our null findings. Trust building must also occur before any meaningful discussion of the goals of care could be achieved, and follow-up time may have to be extended. Toward the end of this study, we developed an extension of our program for patients with limited life expectancy and conservative goals of care. In this extension, reductions in hospitalizations were observed among patients who had multiple goals of care discussions. ${ }^{28}$

Previous studies have shown that readmissions reduced with timely follow up among patients with heart failure. ${ }^{39}$ Our results showed no difference in readmission rate based on whether or not our patients were visited within five days from discharge, but we may have been underpowered to detect this difference. In addition, we may have missed readmissions that occurred before the enrollment visit.

The elements of the CTP were evidence based. Fidelity to program goals improved over time and reached high levels with program maturity. Only $12 \%$ of the patients received all program components at the first home visit. Patients that had all pillars addressed and documented showed a nonsignificant trend toward reduced readmission rates. NPs were given discretion as to how many visits were required to stabilize a patient and achieve program objectives. Heart failure management was driven by protocol with input from cardiology. Medication reconciliation and clinical assessment with action plan were prioritized at the first visit and thus allowed for the completion of other goals at a subsequent visit if time was insufficient. These decisions were deliberated at weekly physician-led multidisciplinary meetings. This variability allowed the team to meet chronic and urgent needs but further confounded the interpretation of our results. One possible way to interpret the lack of significant predictors of success is that through clinical assessment and flexibility, we were able to tailor our program to meet the needs of this complex multi-morbid population.

This study has important limitations. Given that it is a retrospective cohort study, we were unable to include patients who were enrolled but were either readmitted or dropped out before the first program visit. In addition, because of our study's limited sample size and readmission rate, we had limited power to detect other potential predictor variables and test for confounding and interaction. While we included numerous variables in our analyses, we lacked information on mental 


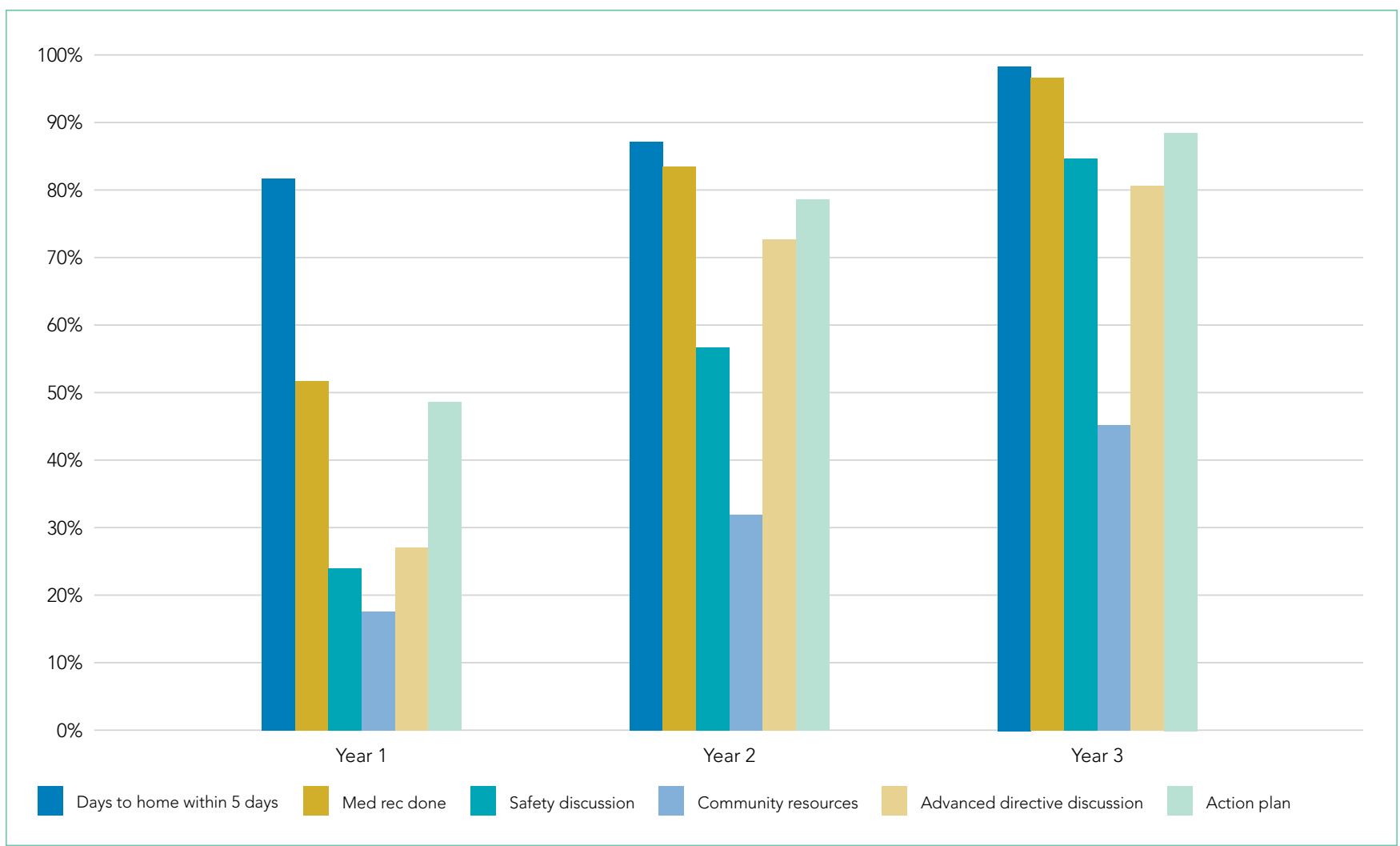

FIG 2. Achievement of Program Fidelity Measures Improved over Time with Increasing Program Maturity.

health and the social determinants of health, which are known to influence readmission risk. ${ }^{40,41}$ Similarly, we lacked patient self-reported measures of health and information regarding caregiver support, which are important. ${ }^{42,43}$ Several of our predictive measures (cognitive impairment, mobility limitations, and program objective completion) were dependent on supplementing billing codes with heterogeneous data abstracted from usual clinical care as opposed to standardized research protocols. Neither method is completely accurate, nor can the combination of the two be assumed to be without inaccuracies. Failure to adequately document the clinical interventions performed by the clinical team is possibly a major confounder as evidenced by the considerable lack of agreement by our trained abstractors in determining whether advance care planning took place. The generalizability of our results is also a concern because the local population is largely white and highly educated, although our experience tells us that many of our program patients have limited means and thus may more closely resemble the general US population. ${ }^{44}$ The strength of our study is that it uses real, practice-based data that can be directly translated to practice.

\section{CONCLUSION}

This study focused on a successful high-intensity CTP. Results showed that compared with patients without dementia, patients with dementia were more likely to avoid hospitalizations as a result of enrollment in the investigated CTP. This study, however, failed to identify specific programmatic components critical for the success of the CTP. These findings support the current hypothesis that multidisciplinary, multimodal, and highly intensive interventions are necessary to care for complex and multi-morbid patients. They also suggest that compared with cognitively functional patients, cognitively impaired patients with conservative goals of care may be more likely to avoid burdensome hospitalizations when provided with early intervention in their home.

\section{Acknowledgments}

B.T. conceived and designed the study, interpreted the data, drafted and provided final revisions to the manuscript. P.Y.T, N.D.S., and J.M.N obtained funding, contributed to the conception and design of the study, analysis, and interpretation of the data, and provided critical revisions to the manuscript. P.A.R., R.G.M, and G.J.H., contributed to the conception and design of the study, analysis, and interpretation of the data, and provided critical revisions to the manuscript. S.M.P. Assisted with data acquisition and interpretation, performed the data analysis, and drafted parts of the manuscript. C.Y.Y.C, L.J.H., A.L, A.C., L.B., and R.H. helped with methodologic questions and data interpretation, and provided critical revisions to the manuscript.

All authors read and approved the final manuscript and the decision to submit the manuscript for publication.

We thank Donna Lawson, RN for her help with data abstraction and Annika Beck and Anna Jones in Mayo Clinic Biomedical Ethics Research Program for her help in preparing this manuscript for publication.

Disclosures: The authors declare no conflicts of interest.

Funding: This publication was supported by the Mayo Clinic, Robert D and Patricia E. Center for the Science of Health Care Delivery (B.T., R.H., R.G.M, L.J.H), by the Extramural Grant Program by Satellite Healthcare, a not-for-profit renal care 
provider (L.J.H., B.T.), and by the National Institute of Health (NIH) National Institute Of Diabetes And Digestive And Kidney Diseases grant K23 DK109134 (L.J.H.) K23DK114497 (RGM) and National Institute on Aging grant K23 AG051679 (B.T.). Additional support was provided by the National Center for Advancing Translational Sciences grant UL1 TR000135. Study contents are the sole responsibility of the authors and do not necessarily represent the official views of $\mathrm{NIH}$.

Funder's Role: The sponsors had no role in the design, execution, or reporting of this study.

Prior Presentations: Part of this data was presented in poster format at the American Geriatrics Society meeting in Washington, DC; 2015.

\section{References}

1. Joynt KE, Jha AK. A path forward on Medicare readmissions. N Engl J Med. 2013;368(13):1175-1177. doi: 10.1056/NEJMp1300122.

2. Epstein AM, Jha AK, Orav EJ. The relationship between hospital admission rates and rehospitalizations. N Engl J Med. 2011;365(24):2287-2295. doi: 10.1056/NEJMsa1101942.

3. Zuckerman RB, Sheingold SH, Orav EJ, Ruhter J, Epstein AM. Readmissions, observation, and the hospital readmissions reduction program. N Engl $J$ Med. 2016;374(16):1543-1551. doi: 10.1056/NEJMsa1513024.

4. Jencks SF, Williams MV, Coleman EA. Rehospitalizations among patients in the Medicare fee-for-service program. N Engl J Med. 2009;360(14):14181428. doi: 10.1056/NEJMsa0803563.

5. Gerhardt G, Yemane A, Hickman P, Oelschlaeger A, Rollins E, Brennan N. Medicare readmission rates showed meaningful decline in 2012. Medicare Medicaid Res Rev. 2013;3(2). doi: 10.5600/mmrr.003.02.b01.

6. Naylor MD, Brooten D, Campbell R, et al. Comprehensive discharge planning and home follow-up of hospitalized elders: a randomized clinical trial. JAMA. 1999;281(7):613-620

7. Takahashi PY, Naessens JM, Peterson SM, et al. Short-term and long-term effectiveness of a post-hospital care transitions program in an older, medically complex population. Healthcare. 2016;4(1):30-35. doi: 10.1016/j.hjdsi.2015.06.006

8. Desai NR, Ross JS, Kwon JY, et al. Association between hospital penalty status under the hospital readmission reduction program and readmission rates for target and nontarget conditions. JAMA. 2016;316(24):2647-2656. doi: 10.1001/jama.2016.18533.

9. CMS. U.S. Centers for Medicare \& Medicaid Services (CMS) measure methodology. https://www.cms.gov/Medicare/Quality-Initiatives-Patient-Assessment-Instruments/HospitalQualitylnits/Measure-Methodology.html. Accessed December 1, 2017; 2017.

10. National Committee for Quality Assurance. All-Cause Readmissions: the Number of Acute Inpatient Stays during the Measurement Year That Were Followed by an Acute Readmission for Any Diagnosis within 30 Days and the Predicted Probability of an Acute Readmission, for Patients 18 Years of Age and Older. Accessed May 18, 2017; 2014.

11. Naylor MD, Hirschman KB, Hanlon AL, et al. Comparison of evidence-based interventions on outcomes of hospitalized, cognitively impaired older adults. J Comp Eff Res. 2014:3(3):245-257. doi: 10.2217/cer.14.14.

12. Le Berre M, Maimon G, Sourial N, Guériton M, Vedel I. Impact of transitional care services for chronically ill older patients: A systematic evidence review. $J$ Am Geriatr Soc. 2017;65(7):1597-1608. doi: 10.1111/jgs.14828.

13. Leppin AL, Gionfriddo MR, Kessler M, et al. Preventing 30-day hospital readmissions: A systematic review and meta-analysis of randomized trials. JAMA Intern Med. 2014;174(7):1095-1107. doi: 10.1001/jamainternmed.2014.1608.

14. Hansen LO, Young RS, Hinami K, Leung A, Williams MV. Interventions to reduce 30-day rehospitalization: a systematic review. Ann Intern Med. 2011:155(8):520-528. doi: 10.7326/0003-4819-155-8-201110180-00008.

15. Takahashi PY, Tung EE, Crane SJ, Chaudhry R, Cha S, Hanson GJ. Use of the elderly risk assessment (ERA) index to predict 2-year mortality and nursing home placement among community dwelling older adults. Arch Gerontol Geriatr. 2012;54(1):34-38. doi: 10.1016/j.archger.2011.02.012.

16. Biehl M, Takahashi PY, Cha SS, Chaudhry R, Gajic O, Thorsteinsdottir B. Prediction of critical illness in elderly outpatients using elder risk assessment: a population-based study. Clin Interv Aging. 2016;11:829-834. doi: 10.2147/ CIA.S99419.

17. Takahashi PY, Haas LR, Quigg SM, et al. 30-day hospital readmission of older adults using care transitions after hospitalization: a pilot prospective cohort study. Clin Interv Aging. 2013:8:729-736. doi: 10.2147/CIA.S44390.

18. Dunlay SM, Pack QR, Thomas RJ, Killian JM, Roger VL. Participation in cardiac rehabilitation, readmissions, and death after acute myocardial infarction. Am J Med. 2014;127(6):538-546. doi: 10.1016/j.amjmed.2014.02.008.

19. Crane SJ, Tung EE, Hanson GJ, Cha S, Chaudhry R, Takahashi PY. Use of an electronic administrative database to identify older community dwelling adults at high-risk for hospitalization or emergency department visits: the elders risk assessment index. BMC Health Serv Res. 2010;10:338. doi: 10.1186/1472-6963-10-338

20. Charlson ME, Pompei P, Ales KL, MacKenzie CR. A new method of classifying prognostic comorbidity in longitudinal studies: development and validation. J Chronic Dis. 1987:40(5):373-383. doi: 10.1016/0021-9681(87)90171-8.

21. Collin C, Wade DT, Davies S, Horne V. The Barthel ADL Index: a reliability study. Int Disabil Stud. 1988;10(2):61-63. doi: 10.3109/09638288809164103.

22. Sulter G, Steen C, De Keyser J. Use of the Barthel index and modified Rankin scale in acute stroke trials. Stroke. 1999;30(8):1538-1541. doi: 10.1161/01. STR.30.8.1538

23. Bohannon RW. Reference values for the timed up and go test: A descriptive meta-analysis. J Geriatr Phys Ther. 2006;29(2):64-68. doi: 10.1519/00139143200608000-00004

24. Kokmen E, Naessens JM, Offord KP. A short test of mental status: description and preliminary results. Mayo Clin Proc. 1987;62(4):281-288. doi: 10.1016/ S0025-6196(12)61905-3.

25. Folstein MF, Folstein SE, McHugh PR. "Mini-mental state". A practical method for grading the cognitive state of patients for the clinician. J Psychiatr Res. 1975;12(3):189-198. doi: 10.1016/0022-3956(75)90026-6.

26. Galvin JE, Roe CM, Powlishta KK, et al. The AD8: A brief informant interview to detect dementia. Neurology. 2005;65(4):559-564. doi: 10.1212/01. wnl.0000172958.95282.2a

27. Farrell B, Szeto W, Shamji S. Drug-related problems in the frail elderly. Can Fam Phys. 2011;57(2):168-169.

28. Chen CY, Thorsteinsdottir B, Cha SS, et al. Health care outcomes and advance care planning in older adults who receive home-based palliative care: a pilot cohort study. J Palliat Med. 2015;18(1):38-44. doi: 10.1089/jpm.2014.0150.

29. Rao A, Suliman A, Vuik S, Aylin P, Darzi A. Outcomes of dementia: systematic review and meta-analysis of hospital administrative database studies. Arch Gerontol Geriatr. 2016;66(Suppl C):198-204. doi: 10.1016/j.archger.2016.06.008.

30. Gozalo P, Teno JM, Mitchell SL, et al. End-of-life transitions among nursing home residents with cognitive issues. N Engl J Med. 2011;365(13):1212-1221. doi: 10.1056/NEJMsa1100347.

31. Wang SY, Aldridge MD, Gross CP, Canavan M, Cherlin E, Bradley E. Endof-life care transition patterns of Medicare beneficiaries. J Am Geriatr Soc. 2017;65(7):1406-1413. doi: 10.1111/jgs.14891.

32. Pedersen MK, Meyer G, Uhrenfeldt L. Risk factors for acute care hospital readmission in older persons in Western countries: a systematic review. JBI Database System Rev Implement Rep. 2017;15(2):454-485. doi: 10.11124/ JBISRIR-2016-003267.

33. Edwards ST, Saha S, Prentice JC, Pizer SD. Preventing hospitalization with Veterans Affairs home-based primary care: which individuals benefit most? J Am Geriatr Soc. 2017;65(8):1676-1683. doi: 10.1111/jgs.14843.

34. Mitchell SL, Palmer JA, Volandes AE, Hanson LC, Habtemariam D, Shaffer ML. Level of care preferences Among nursing home residents With advanced dementia. J Pain Symptom Manage. 2017;54(3):340-345. doi: 10.1016/j.jpainsymman.2017.04.020.

35. D'Avolio DA, Strumpf NE, Feldman J, Mitchell P, Rebholz CM. Barriers to primary care: perceptions of older adults utilizing the ED for nonurgent visits. Clin Nurs Res. 2013:22(4):416-431. doi: 10.1177/1054773813485597.

36. Brinkman-Stoppelenburg A, Rietjens JA, van der Heide A. The effects of advance care planning on end-of-life care: a systematic review. Palliat Med. 2014;28(8):1000-1025. doi: 10.1177/0269216314526272.

37. Martin RS, Hayes B, Gregorevic K, Lim WK. The effects of advance care planning interventions on nursing home residents: A systematic review. J Am Med Dir Assoc. 2016;17(4):284-293. doi: 10.1016/j.jamda.2015.12.017.

38. Auerbach AD, Kripalani $S$, Vasilevskis EE, et al. Preventability and causes of readmissions in a national cohort of general medicine patients. JAMA Intern Med. 2016;176(4):484-493. doi: 10.1001/jamainternmed.2015.7863.

39. Parrinello G, Torres D, Paterna $S$, et al. Early and personalized ambulatory follow-up to tailor furosemide and fluid intake according to congestion in post-discharge heart failure. Intern Emerg Med. 2013;8(3):221-228. doi: 10.1007/s11739-011-0602-y.

40. Barnett ML, Hsu J, McWilliams JM. Patient characteristics and differences in hospital readmission rates. JAMA Intern Med. 2015;175(11):1803-1812. doi: 10.1001/jamainternmed.2015.4660.

41. Calvillo-King L, Arnold D, Eubank KJ, et al. Impact of social factors on risk of readmission or mortality in pneumonia and heart failure: systematic review. $J$ Gen Intern Med. 2013;28(2):269-282. doi: 10.1007/s11606-012-2235-x.

42. Rönneikkö JK, Mäkelä M, Jämsen ER, et al. Predictors for unplanned hospitalization of New Home care clients. J Am Geriatr Soc. 2017;65(2):407-414. doi: 10.1111/jgs. 14486

43. Hasan O, Meltzer DO, Shaykevich SA, et al. Hospital readmission in general medicine patients: a prediction model. J Gen Intern Med. 2010;25(3):211219. doi: $10.1007 / \mathrm{s} 11606-009-1196-1$

44. St Sauver JL, Grossardt BR, Leibson CL, Yawn BP, Melton LJ, 3rd, Rocca WA. Generalizability of epidemiological findings and public health decisions: an illustration from the Rochester Epidemiology Project. Mayo Clin Proc. 2012;87(2):151-160. doi: 10.1016/j.mayocp.2011.11.009. 